\title{
Space optics contributions by the College of Optical Sciences over the past 50 years
}

\author{
James B. Breckinridge $*^{\mathrm{a}}$ and Peter Smith ${ }^{\mathrm{b}}$ \\ ${ }^{a}$ Graduate Aeronautical Laboratory, M/S 150-50 Firestone, Caltech, 1200 E. California Blvd. , \\ Pasadena, CA., 91125; ${ }^{\mathrm{b}}$ Lunar and Planetary Laboratory, University of Arizona, Tucson, AZ. \\ 85721
}

\begin{abstract}
We present a review of the contributions by students, staff, faculty and alumni to the Nation's space program over the past 50 years. The balloon polariscope led the way to future space optics missions. The missions Pioneer Venus (large probe solar flux radiometer), Pioneer 10/11 (imaging photopolarimeter) to Jupiter and Saturn, Hubble Space Telescope (HST), and next generation large aperture space telescopes are discussed.
\end{abstract}

Keywords: Pioneer 10/11, Imaging, Photopolarimetry, Pioneer Venus, Jupiter, Saturn, Venus, Radiometry, balloon polariscope and space telescopes

\section{INTRODUCTION}

The successful development of rocket engines during WW2 inspired the image of space telescopes and the exploration of the solar system. The May 29, 1944 issue of Life Magazine (p. 78) shows the first science based drawings of what a man walking on a planetary surface might see. Made by Chesley Bonestell these images inspired generations of astronomers, geologists and the general public. In 1946 Professor Lyman Spitzer of Princeton University proposed the construction of a space telescope for astrophysics. Thus were planted the seeds of modern space telescopes for planetary science and astrophysics.

The Optical Science Center was founded only 6 years after the Russians orbited Sputnik. In the fall of 1958 President Eisenhower established the civilian space agency: National Aeronautics and Space Agency (NASA). NASA was formed by combining scientific and engineering centers (Ames, Goddard and Lewis) from the National Advisory Committee for Aeronautics (NACA) and the U. S. Army military research labs at JPL in Pasadena and at Redstone Arsenal in Huntsville, Ala. In Alabama, Werner von Braun and his team were developing launch vehicles. The NASA's optics interests include research and engineering in both aeronautics optics and space optics. The agency was chartered to be civilian, not military and placed under Housing and Urban Development, where it resides today. The scientific exploration of space was and remains to this day a major part of its charter.

Shortly after the founding of the agency, NASA created a program for the unmanned exploration of the solar system. Innovative imaging systems, radiometers, spectrometers and image processing became an important part of the space program. In January 1961, President Kennedy challenged the Nation to send a man to the moon! Scientist faced questions like: what is the environment like on the surface of the moon. The Ranger Program had 2 launch failures, three spacecraft failures and a failure of the return beam Vidicon (Television) Camera. Finally, the seventh Ranger spacecraft made it with the cameras running impacted the surface of the moon. Ranger 7, 8 and 9 were successes. Images of the surface of the moon just before impact were sent back. The technologist who built these vidicons for an early balloon-imaging program at OSC/LPL moved to JPL to build these vidicons for the Ranger program.

Fifty Years of Optical Sciences at The University of Arizona, edited by Harrison H. Barrett,

John E. Greivenkamp, Eustace L. Dereniak, Proc. of SPIE Vol. 9186, 918606

(C) 2014 SPIE - CCC code: $0277-786 X / 14 / \$ 18 \cdot$ doi: 10.1117/12.2064892 
By 1964 it had become clear that there were four National needs that required innovative optical system design and engineering for aeronautics and space exploration.

- Airborne mapping systems, reconnaissance cameras and airborne imaging spectrometers for intelligence gathering required new technologies, engineering \& methodologies and calibration techniques. Cameras developed for manned airplanes could not be used for unmanned spacecraft.

- Astronomers realized that new astronomy required new telescopes and instruments. Instruments on rockets and balloons had shown astronomers a whole new universe.

- The earth remote sensing communities had visions to use satellite cameras for land management, crop assessment, atmospheric science, watershed management, forestry and many others.

- The public expressed great interest in planetary exploration. Optical instruments, imagers and spectrometers were needed to explore the solar system from the UV through the IR.

Throughout the 60's the space race was very real. After the US successful moon landing in 1969 and the additional Apollo missions to explore the moon, the space race continued. In the 1970's the manned program to the moon was replaced by the successful space shuttle program. Remote sensing of the solar system with the vision, in some people's minds of someday colonizing other planets in our solar system was and is today very real.

Over the past 50 years research and development at the College of Optical Sciences has paved the way for new instruments, telescopes and techniques. Here we will review OSC's contributions in planetary exploration and astrophysics within the framework of several activities: 1 . The balloon polariscope program led by Tom Gehrels; 2 . The first spacecraft to Jupiter and Saturn, Pioneer 10 and 11 imaging photopolarimeter led by T. Gehrels and M. Tomasko; 3. The Pioneer Venus solar flux radiometer, led by Bill Wolf and Jim Palmer; 4. Hubble Space Telescope failure review board and wavefront correction. Aden Meinel was one of the pioneers in large space telescopes for astronomy. Over the past 50 years more than $150 \mathrm{OSC}$ alumni are estimated to have spent their careers designing and building space optics for astrophysics, earth, planetary and now exoplanetary science.

\section{BALLOON TELESCOPES - BALLOON POLARISCOPE}

The cost of space missions both in dollars and the extreme length of time it took before scientific results were obtained. The value to science above the Earth's atmosphere and of space missions needed to be demonstrated. Princeton astronomers launched several balloon borne telescopes (Stratoscope project) to operate in the dry excellent seeing provided by the upper stratosphere to demonstrate the value of space science. The optics for this telescope were developed by a faculty member before he came to OSC and the designs became the subject of classroom examples.

OSC had its own balloon project led by Tom Gehrels who had a joint appointment across OSC and the Lunar and Planetary Laboratory. Between 1965 and 1970 a 71-cm Cassegrain reflecting telescope system, called the Balloon Polariscope was flown to altitudes up to $36.6 \mathrm{~km}$ four times for polarimetry at wavelengths between 0.2 and 0.3 microns of stars and planets. At this altitude, residual Ozone absorption was discovered to be on the order of 1 magnitude per air-mass. The gondola weighed $730 \mathrm{~kg}$ at lift off and it carried, in addition to the telescope, two vidicons cameras, command and telemetry rf equipment and a star tracker. Polarization measurements were made at $225 \mathrm{~nm}$-wavelength. The Balloon Polariscope measured the polarization at Venus, at a 97.9-degree phase angle to be $22 \%$. For Mars the system discovered that at 25.4-degree phase angle Mars showed 6\% polarization. Balloon Polariscope discovered that the cause of interstellar polarization is not metallic or graphitic grains, but some undefined phenomenon. This pioneering work demonstrated the value of polarization measurements from above the atmosphere and supported OptSci graduate students in a variety of roles.

\section{PIONEER 10/11 IMAGING PHOTOPOLARIMETER}

Faculty and students at the College pioneered the use of photo-polarimetry to identify surface materials on the Earth's moon, the surface of other satellites in the solar system and the atmospheres of Venus, Jupiter and Saturn using the TBD $\mathrm{cm}$ telescope on MT. Lemon. It was this broad experience that led NASA to assign the College to design, build, calibrate, operate and interpret the data from an Imaging Photo Polarimeter (IPP) at Jupiter and Saturn. The IPP had begun many years before under the guidance of Dr. Tom Gehrels, who later became the Principal Investigator. He had been studying the polarization of light reflected from solar system objects and realized that much of noise on his signal came from the Earth's atmosphere. In addition important measurements 
needed to be taken out side the transparent windows in our atmosphere. A platform outside of the atmosphere was essential and even better was the prospect of a near encounter with Jupiter and Saturn. Partnering with Dr. Samuel Pellecori, a graduate of OSC, they designed the IPP and proposed it for the Pioneer mission; it was selected for flight from among 150 instrument proposals in 1970. The Pioneer 10 was launched from Cape Canaveral on March 3, 1972 and closest approach to Jupiter was on December 4, 1973. This became the first spacecraft to successfully traverse the asteroid belt and enter the Jovian environment. Pioneer 11 shortly followed on April 6, 1973 and reached Jupiter on December 2, 1974. Pioneer 11 used the gravitational field of Jupiter to "slingshot" the spacecraft for a near encounter of Saturn.

Pioneer 10 was the first spacecraft to fly through the asteroid belt, first to fly close to Jupiter and first to cross Neptune's orbit on a course to leave our solar system. In 1983, Pioneer 10 became the first spacecraft to travel past the orbit of the most distant planet, Neptune. Until Voyager 1 exceeded its distance from Earth on February 17, 1998, Pioneer 10 was the most distant human-made object.

Pioneer 10 and Pioneer 11 were two spacecraft managed out of AMES research center. NASA issued a call for proposals and Tom Gehrels responded with a proposal to build two instruments (one for each spacecraft) to create images of the planet Jupiter and its Satellites (Pioneer 10) and images of both Jupiter and Saturn (Pioneer 11). In addition to imaging these instruments contained a Stokes polarimeter at the focal plane. The two instruments were photopolarimeters.

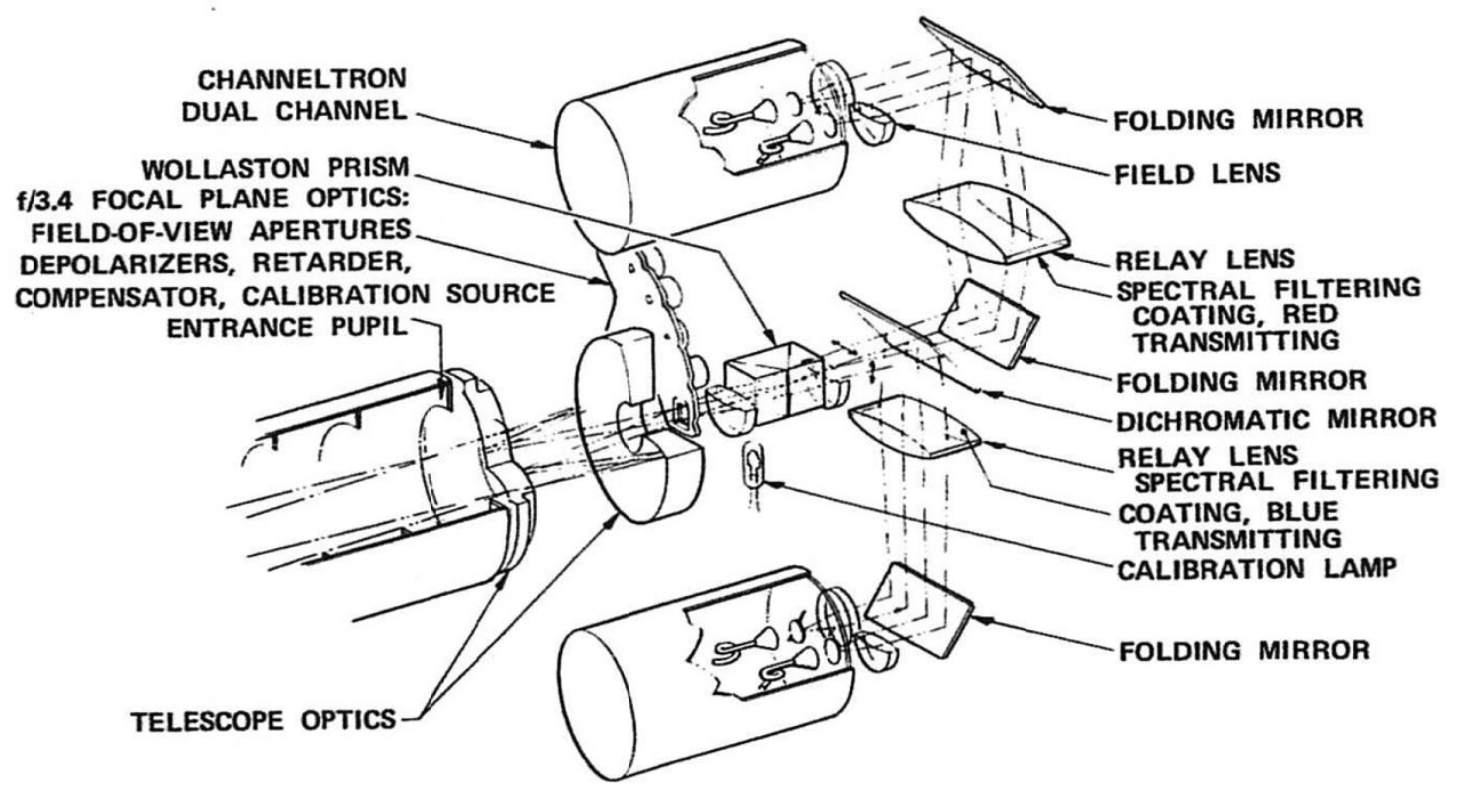

Figure 1. Exploded view of the $2.5 \mathrm{~cm}$ clear aperture imaging photopolarimeter (IPP) on Pioneer 10 and 11 showing details of the optical system. Figure from NASA publication SP 282.

The drawing in figure 1 shows an exploded view of the IPP. Light enters the system traveling from the lower left to right through an $\mathrm{F} \#=3.4$ Cassegrain telescope. The focal plane contains a filter mechanism so the user can choose between field of view apertures which contain a depolarizer, retarder plate, and optics to select a calbration lamp. A Wollaston prism is placed after the field lens to create two images, one each in orthogonal polarization. A dichromatic beamsplitter separates out the two wavelengths of the instrument. Radiation passes through spectral filtes and passess into two dual channel channeltron electron multipliers where the intensity of the radiation is recorded. The angular FOV of the instrument was 0.5 by 0.5 milliradians.

A single object space pixel was divided into four pixels and passed to 4 detectors. As the light enters the system from the planet, the irradiance in the pixel is divided into two orthogonal polarization states using the Wollaston prism. Then light from each of these two polarization states is further divided into two wavelength bandpasses to give 4 beams falling on 4 detectors.

During the flybys, objects were imaged through a 25 -mm-diameter lens onto a small aperture and then divided into $\mathrm{S}$ and $\mathrm{P}$ polarization components through a Wollaston prism before being finally split apart through 
dichroic filters into two wavelength bands red $(640 \mathrm{~nm})$ and blue $(440 \mathrm{~nm})$. Thus, four detectors were needed to capture the data set. Each one is essentially a one-pixel camera. An image was created using a spin-scan technique taking advantage of the spacecraft's spin stabilization and adjusting the clock angle (the angle between the lens optical axis and the spacecraft spin axis) for pointing. It was naturally important to limit the data-taking period to the proper portion of the spin cycle. Sometimes the clock angle was stepped to create an image, other occasions would allow the relative motions of the spacecraft and object to scan the image.

Unlike a normal framing camera every pixel is exposed at a different time, being on a moving platform the geometry of the images was often severely distorted especially during the high-speed, close flybys. We called the images banana plots and developed methods for proper geometric reconstruction. However, there is a great advantage for imaging with a single pixel — all parts of the image have the same radiometric properties, there are no bad pixels or flat fielding effects. Furthermore, once the data is returned to Earth, images are created by reversing the process. Photographic film or paper is attached to a drum is slowly rotated while a light source modulated by the pixel intensity is scanned along its length. The small group who were tasked with converting digital tapes containing the image data to hardcopy consisted of Ed Beshore, Joe Gotobed, Rob Kingston, and Rod Norden. The photographic processing went though Gil McLaughlin's photographic studio and keeping us all on budget was Charlie Blenman.

The photometry and polarimetry was analyzed across the street at the Lunar and Planetary Lab by Tom Gehrels' team. The primary scientist was Martin Tomasko along with Lyn Doose and supported by co-investigator Charles KenKnight, Del Castillo, and Lonnie Baker. The high phase angle observations not possible from Earth gave important clues to the structure of the Jovian atmosphere.

Several degrees were earned by using the Pioneer Jupiter data. I published a paper in Icarus measuring the diameters of the four Galilean Satellites with an accuracy of about 0.1 pixels. This paper was later accepted as a Masters thesis. Lyn Doose wrote his doctoral dissertation on the observations of Jupiter's famous great red spot. Cliff Stoll also did a thesis on the photometry of the belts and zones the make up the banded appearance of Jupiter. Robert West, now at JPL, also used some of the Pioneer data in his dissertation.

Figure 2 shows a photograph of the Pioneer 10/11 imaging photopolarimeter with its conical shaped straylight collar pointing to the right. The optical system with detectors shown in Fig 1 was packaged in the flat cylinder shaped structure shown with the white (for thermal control) band. The analog signal amplifiers, A/D's and signal conditioning electronics are inside the black box shown in the upper left. The scale shown in the photograph is in units of inches.

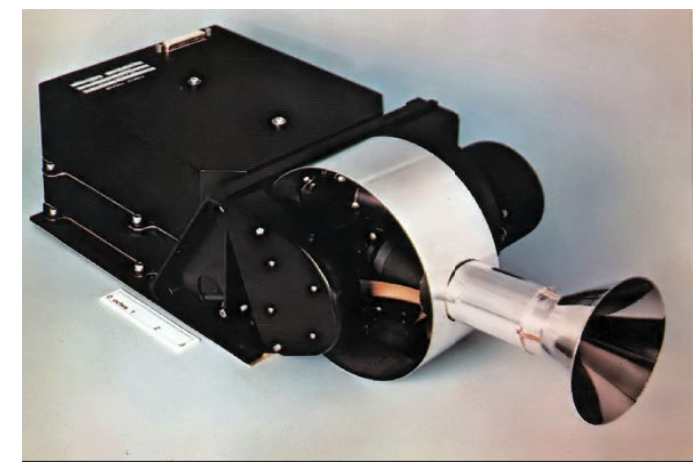

Figure 2 shows a photograph of the outside of the grapefruit-sized $2.5 \mathrm{~cm}$ clear aperture IPP instrument with its tapered stray-light collar pointing to the right. See the scale dimensions at the left. Figure from NASA publication SP 282

Figure 3 contains an illustration of how the IPP instrument recorded images, 4 pixels at a time. The spinning spacecraft provided scans across the planet and the instrument was nodded plus minus 7 degrees to provide a crosstrack scan. 


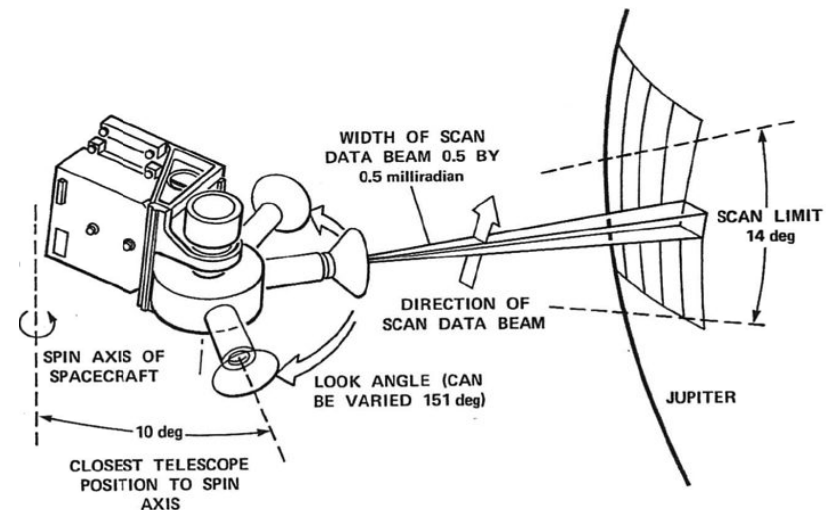

Figure 3 shows a drawing of the exterior of the IPP during a data recording operation, scanning its FOV over the cloud tops of Jupiter. Figure from NASA publication SP 282.

In figure 3 shows the IPP mounted in relation to the verticle spin axis of the space craft. The look angle was adjusted and the instrument nodded upand down to trace out slices across the planet.

During the Pioneer 10 and 11 misions to Jupiter the light-travel time between the Earth and the planet was 46 minutes. Clearly the imaging system needed to be designed to be fully autonomous during the encounter. The rate at which images were reorded by the IPP ment that during operation of the system many images at anyone time were stacked up across space moving from Jupiter to the Earth. During the data downlink there could be as many as 60,000 pixels spaced out between Earth and Jupiter or about 10,000 km/pixel. The illustration in Figure 4 shows a series of image packets propagating at the speed of light from the planet Jupiter to earth during the IPP imaging phase.

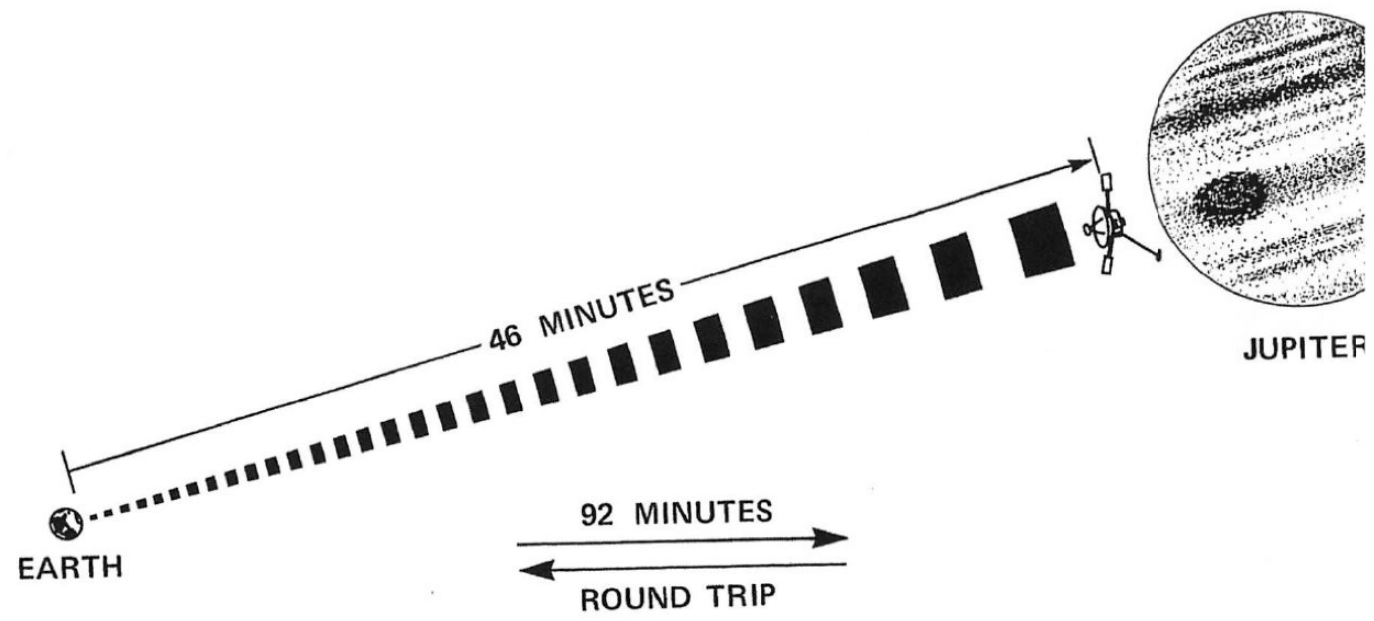

Figure 4 shows IPP images stacked up across space as they travel the 46-minute interval from Jupiter to Earth. Figure from NASA publication SP 282.

Figure 3 showed how images were built up from scans. The task looks far easier that it was. In many cases very small motion of the spacecraft between scans and timing errors would cause the scans not to line up properly. Software to cross-correlate scans was necessary to shift one scan relative to another and build up the image. An example of one of the best images of Jupiter's red spot was recorded by IPP on Pioneer 11, when it flew beneath Jupiter to gain the gravitational assist necessary to get to Saturn almost 5 years later. This image is shown in figure 5 below. 


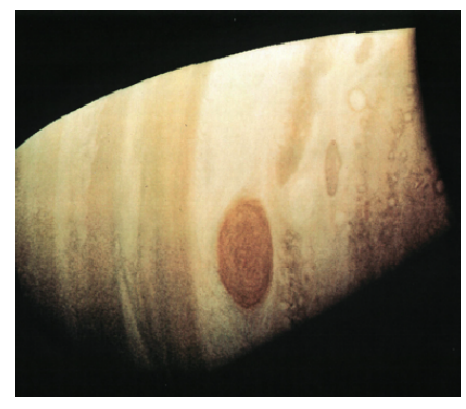

Figure 5 shows the signals from series of scans by the IPP system on Pioneer 11 process to reveal an image Jupiter's great red spot. Figure from NASA publication SP 282.

In order for the mission to reach some of the science objectives other than those of IPP, the Pioneer 10 spacecraft was in an orbit that took it away from other planets after its encounter with Jupiter. Pioneer 11, however, passed beneath Jupiter in just the right orbit to continue over to the planet Saturn to continue its role of imager and photopolarimeter. Figure 6 below shows the path of Pioneer 11 from Earth (launch Apr 1973) to Jupiter (Dec 1974) and then onto Saturn (Sept 1979).

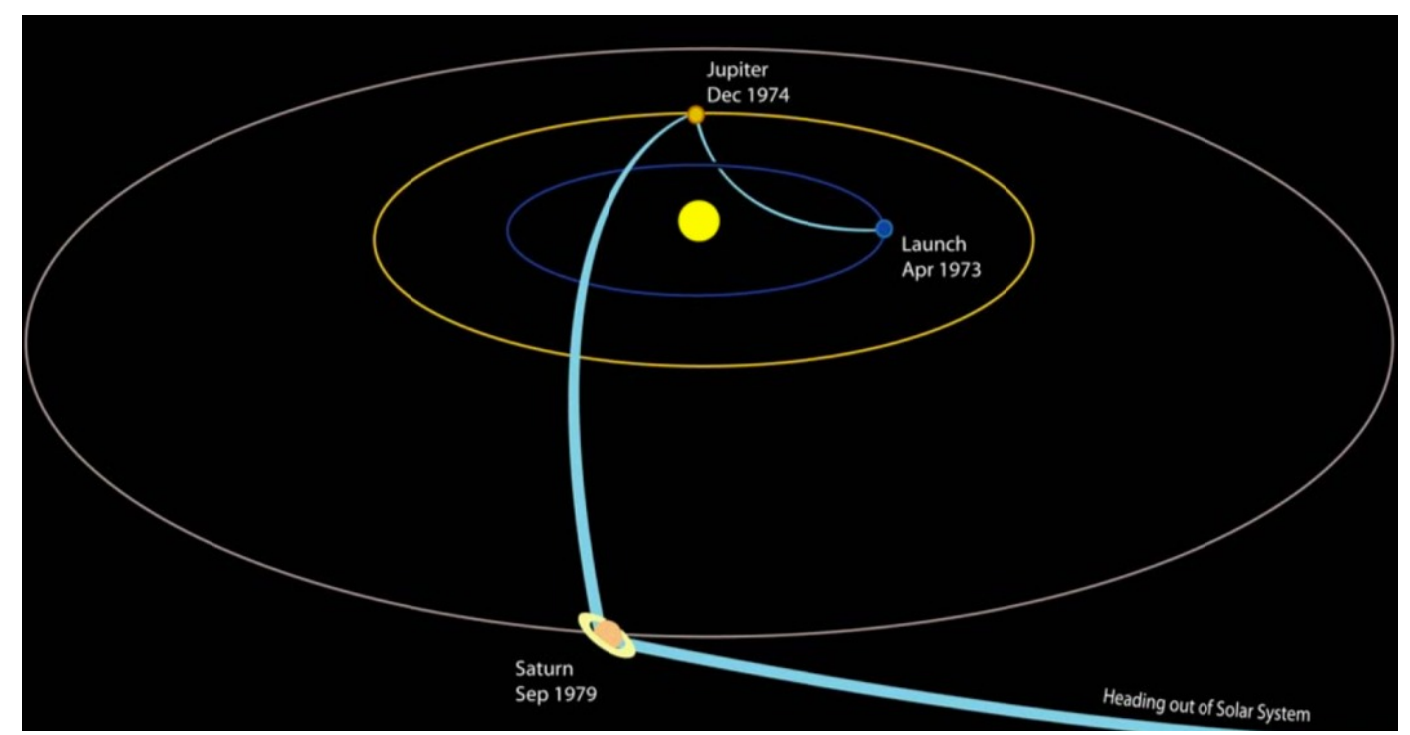

Figure 6 shows the trajectory of Pioneer 11 as it traveled from the Earth at launch April 1973 to pass beneath Jupiter, up and over the plane of the ecliptic to orbit down on Saturn and then accelerate out of the solar system. Figure from NASA publication SP 282

The OSC IPP recorded images of Saturn during the flyby of Sept 1979. The highest resolution image is shown in figure 7 below.

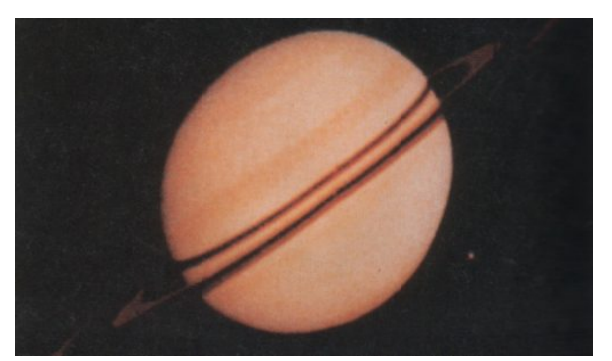

Figure 7 shows the highest resolution image recorded by the IPP of the planet Saturn with its satellite Titan shown in the lower right. Figure from NASA publication SP 282 
Many discoveries were made using the IPP instrument A few of these are listed in table 1, below.

Table 1 provides a list of the discoveries made by Pioneer 11 IPP during its encounter with Saturn.

- New ring discovered which has an optical depth of less than $2 \times 10^{-3}$ and is located at $\mathrm{R}_{\text {Saturn }}$ of 2.33 .

- Measured the width of the Encke Gap to be $876 \mathrm{~km}$

- The mean particle size in the rings is less that 15 meters

- New satellite designated 1979S1 was discovered

- The equatorial radius of the planet was measured to be $60,000+-500 \mathrm{~km}$.

- Ratio of the polar to the equatorial radius is 0.912 .

These images appear rather primitive by today's standards given the results of the Voyager and Galileo imaging and imaging spectrometry missions. But it must be remembered that the Pioneer flybys predated Voyager by over 6 years and Galileo by about 24 years. Without the very exciting scientific data and IPP images from the Pioneer 10/11 spacecraft there may have been no Voyager and Galileo. The College of Optical Sciences contribution to imaging the giant planets provided inspiration to future generations of planetary scientists and space optics scientists and engineers.

\section{PIONEER VENUS: THE LARGE PROBE SOLAR FLUX RADIOMETER}

The Venera series of probes were developed by the Soviet Union between 1961 and 1984 to gather data from the planet Venus. Ten probes successfully landed on Venus and transmitted data from the surface. Venera 7 was the first man-made device to make a soft landing on another planet on December 15, 1970 and transmit data from the surface. This lander found the atmospheric pressure to be $\sim 90$ atmospheres and a surface temperature of $465^{\circ} \mathrm{C}$ $\left(869^{\circ} \mathrm{F}\right)$. Planetary scientists in the US were eager to understand this very high surface temperature, given the extent of the cloud cover and, NASA issued a call for proposals for instruments on a proposed Pioneer Venus mission.

Professor Bill Wolf and a team responded to the call with a plan to design, build, test and calibrate a solar flux radiometer instrument. M. G. Tomasko was the science principal investigator. The scientific questions to be answered by the Pioneer Venus solar flux radiometer are given in Table 2 below.

\section{Table 2 gives a list of scientific questions for the Solar Flux radiometer.}

- Determine the deposition of solar energy in the atmosphere of Venus between $67 \mathrm{~km}$ and the surface

- Measure upward and downward fluxes and radiances with altitude resolution of several hundred meters.

- Where in the deep atmosphere is energy being absorbed?

- Explain the very high surface temperatures $\left(460^{\circ} \mathrm{C} @ 90-\mathrm{Atm}\right)$ measured by the Russian Venus landers Venera

\section{Table 3 gives the attributes of the instrument}

- Size of a Grapefruit

- Five channels using CdS detectors, quartz light pipes and spun as it fell through the atmosphere of Venus

- Measured atmospheric radiance over a spectral range 400 to $1,800 \mathrm{~nm}$ in five windows as a function of altitude

- Detectors maintained at $30^{\circ} \mathrm{C}$ with a phase change material.

- Detector out puts were processed with logarithmic trans-impedance converters and digitized with an 11-bit $\mathrm{A} / \mathrm{D}$ converter

- The serial output data averaged $20 \mathrm{~b} / \mathrm{sec}$, including house keeping such as

- Sync, spin period, sample timing and measurement mode 
On December 9, 1978, the Pioneer Venus Large Probe entered the atmosphere of Venus. First it decelerated using its aeroshell, thereupon, it deployed a parachute and slowly descended through the thick atmosphere to the superheated surface. On board was an experiment named the Large Probe Solar Flux Radiometer (LSFR), an experiment developed at the UA jointly between the Lunar and Planetary Lab with Dr. Martin Tomasko as the PI and the Optical Sciences Center where co-investigator Prof. William Wolfe contributed to the optics and the calibration.

The scientific goals of the instrument were to determine where in the deep atmosphere solar energy was being absorbed to explain the high temperatures that had been measured by Russian probes. To accomplish this goal, three upward looking channels were spaced at specific Gaussian angles so that the downward solar flux could be integrated. Two downward looking channels were used to retrieve the upward flux. The difference between the up and down fluxes, or the net flux, was a measure of the total heating below the altitude of the measurement. The slope of the net flux therefore constrained the altitudes where heating occurred. Balancing the heating with the thermal radiative cooling was an additional calculation that explained the temperature profile and could constrain the mixing ratio of water in the atmosphere.

The amazing result of the experiment is that globally only about $2.5 \%$ of the incident sunlight is absorbed in the ground. Even so, the opacity of the atmosphere in the infra-red caused by $\mathrm{CO} 2$ plus a small amount of water combined with the thick clouds traps this heat and raises the temperatures near the surface to $462^{\circ} \mathrm{C}\left(863^{\circ} \mathrm{F}\right)$.

Early in the development cycle in 1973-4, James Palmer filled the role of chief engineer working with the science team to finalize the opto-mechanical design. This was no easy task given to extreme environmental conditions that Venus provides. His work on the design and calibration of the LSFR under the guidance of Prof. Wolfe led to his dissertation that was completed in 1978.

Others who contributed to the design and construction of the instrument were Dr. Arthur Clements, Arthur G. Bell, Charles Blenman, Lang Brod, Alan Holmes, Roger Kinnard and Even Rosen. Supporting Dr. Tomasko were Dr. Lyn Doose and Peter Smith. One of the major challenges facing the team was the design and construction of the 5 light pipes that transferred the light deep within the instrument where the detector array was cooled by a phase change material. These tiny glass tubes with field stops deposited on their ends were made in the OSC optics shop. 


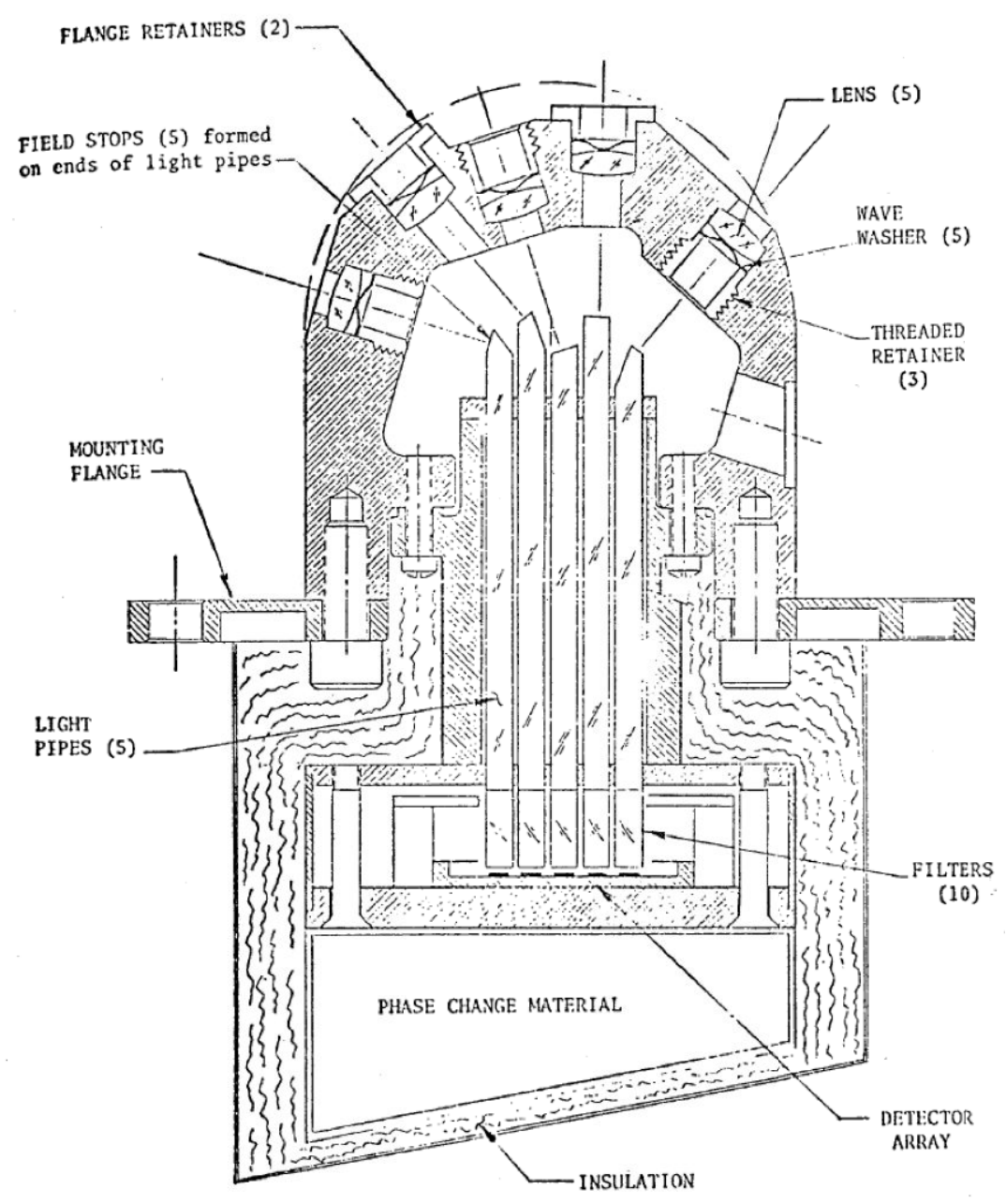

Figure 8. Drawing of the solar flux radiometer built by Prof. Wolfe. Figure from dissertation by James M. Palmer.

Figure 8 shows a drawing of the OSC solar flux radiometer that went to Venus. Radiation entered the system through the 5 lens-windows across the thick, machined-steel dome shaped top. The instrument operates in 1-atm, but the environment outside is 90 -atm and almost $500^{\circ} \mathrm{C}$. Radiation entered each of the 5 channels through sapphire windows mounted in the walls of the pressure chamber. Radiation then passed through a quartz lens and object space was focused onto the top of each of the five light pipes. Each of the five light pipes transmitted the radiation to the detector array at the top of the cooling chamber, which used a phase change material to maintain $30^{\circ} \mathrm{C}$ in the presence of the $460^{\circ} \mathrm{C}$ Venus environment. 


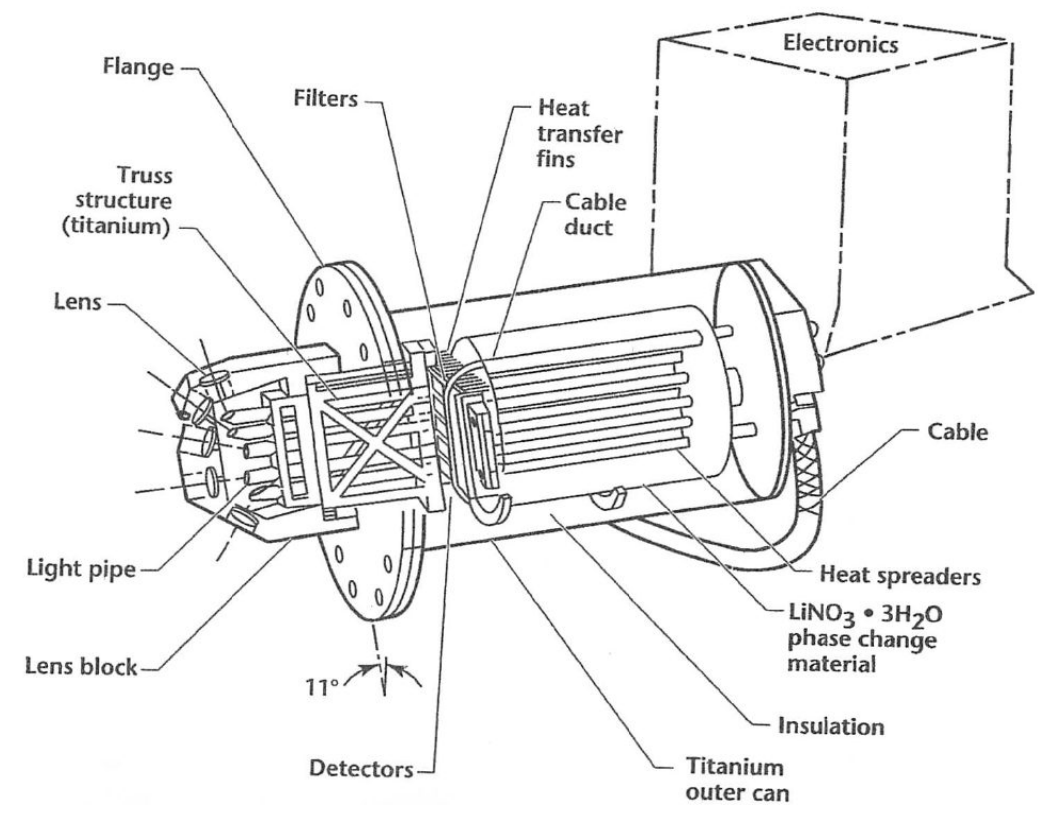

Figure 9 view of the large probe solar flux radiometer built at OSC for the Pioneer Venus mission. Figure from ref [3]

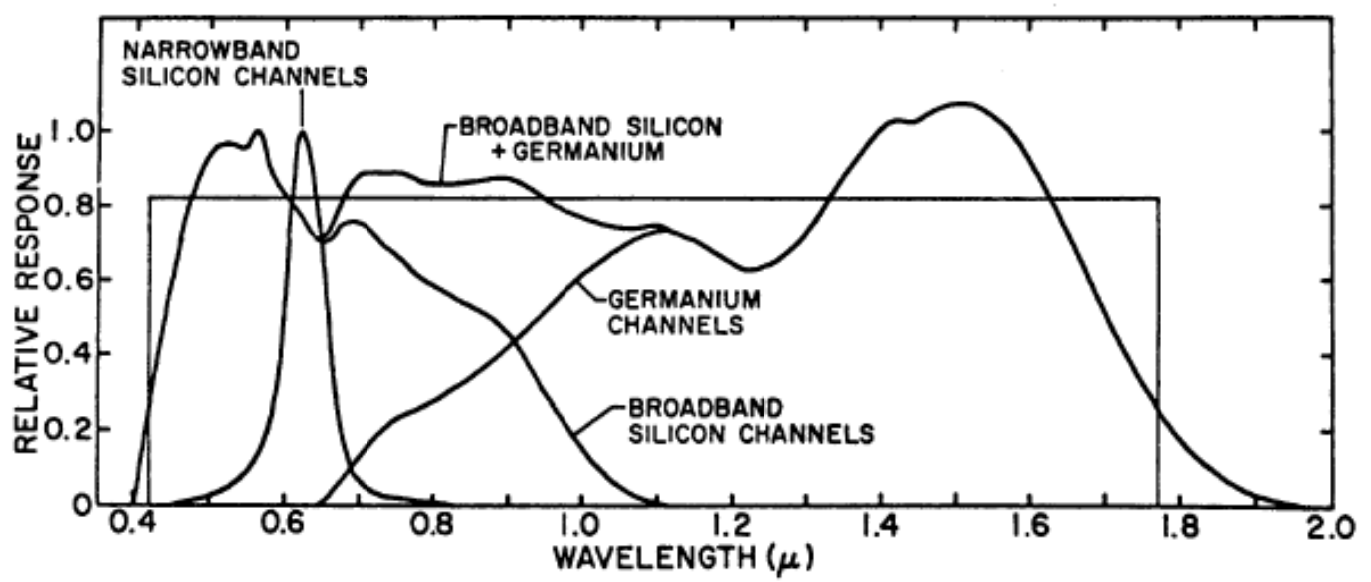

Figure 10 gives the relative response of the system as a function of wavelength for the 5 channels shown in Figure 8 . Fig from ref [3]

\section{OTHER MISSIONS}

In the late eighties, Martin Tomasko and William Wolfe began work that led to proposing a Descent Imager/Spectral Radiometer (DISR) for the Huygens Probe destined to enter Titan's atmosphere and parachute to the surface in January 2005. The probe, built in Europe by the European Space Agency, was carried to the Saturnian system aboard the CASSINI spacecraft and the spacecraft both released the probe on target for the Titan encounter and acted as the communications relay as it flew by the satellite. This long-term project required an extremely complex opto-mechanical and electrical design and Tomasko sought the help of Lockheed Martin in Denver to take responsibility for the design and construction of the instrument.

The sensors for the instrument were provided by European colleagues in Germany (CCD focal plane array) and France (IR senor package). These partnership proved very valuable and helped in proposing future missions. In fact, the German CCD sensor and some of the optical designs became the basis for a Mars Pathfinder camera called 
IMP and led by Peter Smith. The proposal was accepted in 1993 and the mission returned data from the surface of Mars starting on July 4, 1997. The public found this mission very exciting as a small rover called Sojourner was driven from rock to rock exploring the alien landscape.

A series of missions to Mars led by Smith followed but there was little OSC participation. One student, Brant Bos, received his $\mathrm{PhD}$ in Optical Sciences by analyzing the performance of a Robotic Arm Camera (RAC) that became the basis for his long career working with the James Webb Space Telescope at Goddard Space flight Center.

Recently, after 2 failed proposal attempts, LPL, first led by Michael Drake and now led by Dante Lauretta after Drake's passing, a third proposal won a New Frontiers mission to return a sample from an asteroid. The mission, named OSIRIS-REx, is set to launch in 2016 and approach asteroid Bennu in 2019 grabbing a small sample and returning it safely to Earth in 2023. The UA proposed to build the camera system (OCAMS) with the largest element an 8" telescope being designed and built at OSC. Early in the partnership Jim Burge and Marty Valente led the OSC effort, now Rongguang Liang is taking over as science lead. As of this writing, the telescope, called PolyCam, is being tested as an engineering model. Soon the flight model design will be released for construction and testing before delivery in the summer of 2015.

\section{HUBBLE TROUBLE}

Several faculty and alumni were responsible for the successful repair of the Hubble Space Telescope. In 1990, when the failure was announced, Bob Shannon was appointed to the 5-person NASA failure review board along with OSC alumnus Jim Breckinridge in the role of technical advisor to Lew Allen (chair of the committee) and Bob Parks. Aden and Marjorie Meinel served on the science committee charged with creating innovative solutions. The construction of the new Wide Field / Planetary Camera and the COSTAR instrument had many alumni participating.

\section{THE EXOPLANET PROGRAM}

Aden Meinel, after a full career and retirement from the U of A, joined NASA's Jet Propulsion Laboratory (JPL) in 1983 to work on concepts for a 50-meter-diameter submillimeter, segmented space telescope. His work laid the foundation for today's James Webb Space Telescope. In 1986 JPL director Lew Allen Jr. asked Aden for his ideas on future missions for NASA, we concluded that although extremely difficult, the characterization of exoplanets using space telescopes was feasible. Those efforts became the NASA exoplanet program today.

Today, OSC faculty member Olivier Guyon and his group of students are leading both ground-based and spacebased efforts to characterize exoplanets. Prof Guyon, invented a new form of coronagraphic method to characterize exoplanets, called phase induced amplitude apodization (PIAA). The PIAA system, implemented on the Subaru Telescope in Hawaii has revolutionized observational exoplanet science. Prof Guyon currently serves on the AFTA WFIRST-2.4 coronagraph science definition team.

Many alumni of OSC are currently active in exoplanet science and technology.

\section{THE FUTURE OF LARGE SPACE TELESCOPES}

Both astrophysics and exoplanet science require very large aperture telescopes. It is clear that to achieve larger telescopes in space will require multiple launches, robotic assembly, alignment and calibration in space. Technologies and missions to demonstrate these capabilities will occupy the next generation of space telescope optical engineers. The image shown in the Figure 11 below shows a vision for one possible assembly architecture. 


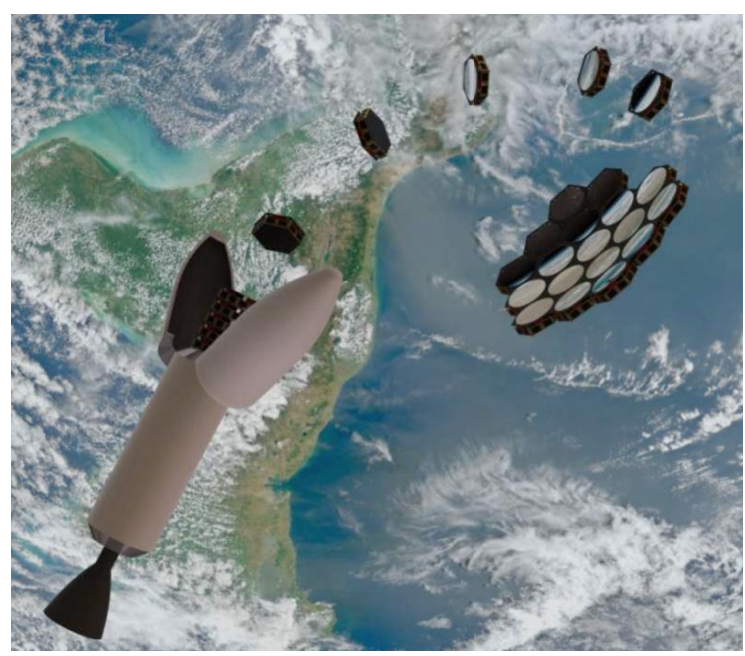

Figure 11. Vision for a possible architecture for assembling the next generation space telescope [4]

\section{SUMMARY}

For 50 years OSC and its alumni have played a role in the design and development of optical telescopes and instruments for planetary, earth, solar, and astrophysics space science. Strong leadership in optical design, engineering, image processing, radiometric calibration and innovative concepts enables the College of Optical Sciences to pave the way into the future for space science.

\section{REFERENCES}

[1] Fimmel, R. O., Swindell, W., Burgess, E., [Pioneer Odyssey], Scientific and Technical Information Office, National Aeronautics and Space Administration, US Government Printing Office \# 1976 O -221-621, stock \# 033-000-00662-4, 217 pages. NASA publication SP 282 (1977)

[2] Colin, L., Hunten, D. M., [Pioneer Venus experiment descriptions Space Science Reviews], Springer Link, Vol 20, pp 451-525 (1977).

[3] Fimmel, R. O., Colin, L. Burgess, E., [Pioneering Venus: a planet unveiled] Scientific and Technical Information Office, National Aeronautics and Space Administration, US Government Printing Office, (1995)

[4] Underwood, C., Pellegrino, S. ..... Breckinridge, J. et. al. [Autonomous Assembly of a Reconfigurable Space Telescope (AAReST) - A cubesat/microsatellite Based Technology Demonstrator], AIAA $27^{\text {th }}$ annual AIAA/USU conference on small satellites, paper SSC-VI-5, (2012) 